\title{
Detection of Oligonucleotide Gas-Phase Conformers: H/D Exchange and Ion Mobility as Complementary Techniques
}

\author{
Dorothée Balbeur, ${ }^{a}$ Joëlle Widart, ${ }^{\text {a Bernard Leyh, }}$, Laetitia Cravello, ${ }^{c}$ \\ and Edwin De Pauw ${ }^{\text {a }}$ \\ a Laboratory of Mass Spectrometry, University of Liège, Liège, Belgium \\ ${ }^{b}$ Molecular Dynamics Laboratory, University of Liège, Liège, Belgium \\ ${ }^{c}$ Waters Corporation, Manchester, United Kingdom
}

Gas-phase hydrogen/deuterium exchange of small oligonucleotides ( $\mathrm{dTG}, \mathrm{dC}_{6}$ and $\mathrm{C}_{6}$ ) with $\mathrm{CD}_{3} \mathrm{OD}$ was performed in the second hexapole of a Fourier transform ion-cyclotron resonance (FTICR) mass spectrometer. Ion activation experiments were conducted by accelerating the ions at the entrance of the H/D exchange cell under conditions promoting exclusively collisional isomerization. These experiments allowed us to assess the presence of several conformers, and to probe the height of the isomerization barrier separating these conformers. Ion mobility experiments were also performed. Their results were consistent with the H/D exchange data. A model accounting for the competing isomerization and $\mathrm{H} / \mathrm{D}$ exchange reactions is proposed. Comparing the ion acceleration experiments for $\mathrm{H} / \mathrm{D}$ exchange and for ion mobility reveals that the most compact conformer displays the fastest $H / D$ exchange. This observation shows that H/D exchange and ion mobility provide us with complementary information because hydrogen accessibility and macromolecule compactness are not univocally associated. (J Am Soc Mass Spectrom 2008, 19, 938-946) (c) 2008 American Society for Mass Spectrometry

$\mathrm{T}$ The knowledge and the understanding of biomolecule conformations are important challenges in chemistry. The conformations of biomolecules depend on intramolecular noncovalent interactions. These interactions govern the majority of biological processes (self-assembly, molecular recognition, regulation, transport, etc.) that define the function of the biomolecules.

Hydrogen/deuterium (H/D) exchange combined with mass spectrometry has become an efficient tool for obtaining conformational information about isolated biomolecules in the gas phase [1-18]. The rate of gasphase H/D exchange has been shown to be a function of the reagent used for exchange (nature and concentration), the charge state of the biomolecule, the gasphase basicity/acidity of exchangeable sites, and the internal structure of the biomolecular ions [1-18]. Probing the number of exchanged hydrogens and the $H / D$ exchange kinetics makes it possible to distinguish ion populations and to elucidate their structural and thermochemical features. Gas-phase H/D exchange has been applied extensively for structural studies of proteins and peptides [1-6] and is now also most commonly used for structural characterizations of oligonucleotides [7-18]. Mononucleotides studies have shown

Address reprint requests to Miss Dorothée Balbeur, Laboratory of Mass Spectrometry, University of Liège, 3 Allée de la Chimie Bat. B6c, B-4000 Liège, Belgium. E-mail: dbalbeur@ulg.ac.be that the extent and rate of exchange depend on the nucleobase (nature, space orientation, and gas-phase acidity of the exchangeable hydrogen), and on the position ( $3^{\prime}$ or $\left.5^{\prime}\right)$ and flexibility of the terminal phosphate group [7-11]. Moreover, these studies have been in favor of the relay mechanism [7-11]. The latter was shown not to be relevant for dinucleotides [14]. Furthermore, gas-phase H/D exchange of dinucleotides has been shown to be controlled by hydrogen accessibility rather than by the chemical nature of the heteroatom bearing the exchangeable hydrogen. Studies of larger oligonucleotides have also been published. Gas-phase $H / D$ exchange reactions were performed on DNA duplexes and on the corresponding single strands. They showed that $H / D$ exchange was slower for the duplex than for the single strands $[15,16]$. In a previous study, a quadruplex was shown to undergo very fast $H / D$ exchange compared with its constituent monomer, an unexpected situation for such a compact and rigid structure [17]. Gas-phase H/D exchange of 5-mer phosphorothioate oligonucleotides was also examined; bimodal exchange profiles were observed, suggesting that each ion could adopt more than one gas-phase conformation [18].

The present work focuses on the bimodal H/D exchange of oligonucleotides. A simple interpretation of such a behavior is that one conformer contains exchangeable hydrogens that mostly belong to two 
groups with very different $H / D$ exchange rate constants, the latter being similar within each group. The situation can, however, be more complex. Two or more conformers may be present. If the exchangeable hydrogens of each conformer belong to either of two groups with well-differentiated $\mathrm{H} / \mathrm{D}$ exchange rates and if these two groups behave similarly in all conformers, a bimodal exchange pattern will show up. The probability that two groups of exchangeable hydrogens have close rate constants for all conformers decreases when the number of conformers increases. As a result, the observation of more than two modes in the H/D exchange mass spectrum becomes more probable when there are more than two conformers. Moreover, when several conformers are present, two situations can be met. Either the different conformers interconvert on the spectrometer time scale and at the experimental temperature or they do not. Usual H/D exchange measurements cannot discriminate these two situations. To assign the observed bimodal H/D exchange to the presence of one or several structures, and to decide whether these distinct conformers interconvert or not, ion activation experiment must be performed. For these experiments, the ions are activated before H/D exchange under selective conditions promoting exclusively collisional isomerization.

Another powerful technique for investigating the gas-phase structure of biomolecules is ion mobility coupled with mass spectrometry. The ion mobility of a gas-phase ion is a measure for the drift speed of this ion through a buffer gas under the influence of a uniform or oscillating electric field. Measuring the drift time directly linked to the mobility of an ion yields information about its structure because compact ions, which have necessarily relatively small collision cross sections, move faster than extended ones, which have large collision cross sections. The gas-phase conformations of many oligonucleotides have been investigated by ion mobility: deprotonated mononucleotides [19, 20], dinucleotides [21], trinucleotides [22], deprotonated oligothymidines [23], DNA complexes [24], G-quadruplex $[25,26]$, etc.

Both hydrogen/deuterium exchange and ion mobility spectrometry provide us with information about the structures and relative stabilities of gas-phase conformers. Studies combining these two techniques have illustrated the difficulty to compare results from H/D exchange with ion mobility results because these two methods probe different physical properties of an ion $[5,6]$. In the present work, ion mobility measurements are used to investigate oligonucleotide structures independently of the H/D exchange experiments. As for $\mathrm{H} / \mathrm{D}$ exchange, experiments involving collisional activation of the ions before the mobility drift were performed.

The dTG DNA dinucleotide, the $\mathrm{dC}_{6}$ DNA hexanucleotide, and the $\mathrm{C}_{6}$ RNA hexanucleotide have been selected for this study. Other small oligonucleotides have also been screened. However, among all of them, only dTG and $\mathrm{C}_{6}$ displayed a bimodal H/D exchange. This is the reason why we submitted them to a detailed investigation. $\mathrm{dC}_{6}$, displaying a mono-modal $\mathrm{H} / \mathrm{D}$ exchange, was chosen as a counter-example. No detailed structural study by molecular modeling was carried out in this work for the selected oligonucleotides.

\section{Experimental}

\section{Sample Preparation}

The DNA dinucleotide (dTG), the DNA hexanucleotide $\left(\mathrm{dC}_{6}\right)$, and the RNA hexanucleotide $\left(\mathrm{C}_{6}\right)$ were obtained from Eurogentec (Seraing, Belgium). $\mathrm{CD}_{3} \mathrm{OD}$ was purchased from Euriso-Top (Gif sur Yvette, France). All these compounds were used without further purification. Stock solutions of the oligonucleotides (with precisely known concentration around $400 \times 10^{-6} \mathrm{M}$ ) were prepared in water. Working solutions $\left(1.1765 \times 10^{-5} \mathrm{M}\right)$ were obtained by dilution in water. The injection solutions were prepared by adding the appropriate amount of $\mathrm{MeOH}$ to obtain a $\mathrm{MeOH} / \mathrm{H}_{2} \mathrm{O}$ ratio of $15 / 85$ (vol/ vol) and an oligonucleotide concentration of $1.0000 \times$ $10^{-5} \mathrm{M}$. For the injection solutions of the RNA hexanucleotide, $\mathrm{NH}_{4}$ Ac was added to reduce sodium adducts. The $\mathrm{NH}_{4}$ Ac concentration was equal to $50 \mathrm{mM}$.

\section{Gas-Phase H/D Exchange Coupled with Mass Spectrometry}

All H/D exchange experiments were performed with a 9.4 tesla Apex-Qe FTICR mass spectrometer (Bruker Daltonics, Billerica, MA) in negative ion mode as previously described [14]. Briefly, the oligonucleotide solutions were infused via an external Apollo electrospray ion source at a flow rate of $120 \mu \mathrm{L} / \mathrm{h}$ with the assistance of $\mathrm{N}_{2}$ nebulizing gas. The off axis sprayer was grounded, the end-plate was set to $3 \mathrm{kV}$ and the inlet capillary was set to $3.5 \mathrm{kV}$ for the generation of oligonucleotide anions. $\mathrm{A} \mathrm{N}_{2}$ heated drying gas $\left(250^{\circ} \mathrm{C}\right)$ flow assisted desolvation of ESI droplets. The H/D exchange data were generated from mono deprotonated dinucleotide ions and doubly deprotonated hexanucleotide ions. These ions were stored in the hexapole collision cell for $10 \mathrm{~ms}$ and then trapped in the presence of $\mathrm{CD}_{3} \mathrm{OD}$ from $10 \mathrm{~ms}$ to $300 \mathrm{~s}$ (the ions entered the hexapole in one bunch and no other ion entered during the exchange time). The $\mathrm{CD}_{3} \mathrm{OD}$ pressure inside the collision cell was estimated to be $\sim 1 \times 10^{-3}$ mbar from the measured pressure in the surrounding vacuum chamber, equal to $5 \times 10^{-6}$ mbar [14].

For the ion activation experiments, the ions were accelerated when entering the collision cell. For that purpose, the Col-Cell Trap parameter, which is directly related to the voltage applied on the entrance lens of the hexapole and which usually was equal to $5 \mathrm{~V}$, was increased up to $7.5,10,15$, and $20 \mathrm{~V}$. No fragmentation was observed in this range. It is also essential to notice that ion activation takes place only during a short 
period of time at the entrance of the hexapole collision cell after which they oscillate in this hexapole from 10 $\mathrm{ms}$ to $300 \mathrm{~s}$ for H/D exchange with no further activation. Multiple collisions with $\mathrm{CD}_{3} \mathrm{OD}$ lead then preferentially to deactivation. We shall elaborate on this point in the discussion. The activation and H/D exchange steps are thus by and large time- and space-separated.

\section{Ion Mobility Coupled with Mass Spectrometry}

Ion mobility measurements were made on the Synapt High Definition MS system (Waters Corporation, Milford, MA) [27]. This hybrid quadrupole orthogonal acceleration Time-of-Flight (oa-TOF) mass spectrometer combines high-resolution tandem mass spectrometry with high efficiency ion mobility (IMS) based on gasphase separations. It was operated in negative ion mode and samples were infused via a ZSpray source at a flow rate of $300 \mu \mathrm{L} / \mathrm{h}$. The electrosprayed ions were transferred non-mass selectively through the quadrupole into the IMS enabling Triwave device. The Triwave consists of three traveling wave ion guides (T-Wave). The first, the Trap T-Wave, traps and accumulates ions. These stored ions are then gated into the IMS T-Wave in which the ion mobility separation occurs. The Transfer T-Wave is then used to transport the separated ions into the orthogonal acceleration time-of-flight (oa-TOF) mass spectrometer for mass analysis. The pressure in the Trap and Transfer T-Wave regions was about $2.25 \times$ $10^{-2}$ mbar of Argon and the pressure in the IMS T-Wave was 0.56 mbar of Nitrogen. The RF amplitude used on the IMS T-Wave was $380 \mathrm{~V}$ peak-to-peak, IMS traveling wave velocities of $300 \mathrm{~m} / \mathrm{s}$ were used, and the wave pulse heights were ramped from 8 to $12 \mathrm{~V}$ during the course of a single mobility separation. Recording the temporal arrival profile of ions (the so-called mobilogram) was achieved by synchronizing the oa-TOF acquisition with the gated release of ions from the Trap to the IMS T-Wave. The following sequence has been repeatedly applied to reach an acceptable signal/noise ratio: gated pulse followed by 200 TOF mass spectra with a pusher period of $64 \mu \mathrm{s}$. This leads to an overall mobility recording time of $200 \times 64 \mu \mathrm{s}=12.8 \mathrm{~ms}$.

For several experiments, the $\mathrm{dC}_{6}$ and $\mathrm{C}_{6}$ ions were accelerated in the source by increasing the cone voltage from $20 \mathrm{~V}$ in the other experiments to $50 \mathrm{~V}$ and then to $70 \mathrm{~V}$. No fragmentation was observed in this voltage regime.

Decomposition of ion mobility data were performed using Igor Pro 4.07 (WaveMetrics Inc., Lake Oswego, OR). The data have been fitted to a double Gaussian distribution:

$$
l(n)=\frac{A_{1}}{\sigma_{1} \sqrt{2 \pi}} e^{-\frac{\left(n-n_{1}\right)^{2}}{2 \sigma_{1}^{2}}}+\frac{A_{2}}{\sigma_{2} \sqrt{2 \pi}} e^{-\frac{\left(n-n_{2}\right)^{2}}{2 \sigma_{2}^{2}}}
$$

where $\mathrm{A}_{i}$ is the area under each partial contribution characterized by its center $n_{i}$ and its standard deviation $\sigma_{i}$.
It is essential to notice that the ion acceleration experiments for H/D exchange and ion mobility may be compared. The experimental conditions have been selected such that the internal energy transferred upon collisions does not reach the onset of the fragmentation channels: only the low-energy isomerization channel is open in both experiments, even though they cover different time-scales.

\section{Results}

\section{H/D Exchange}

As the $H / D$ exchange reaction time increased from 0.01 to $300 \mathrm{~s}$, an increase of the number of exchanged hydrogens $[\mathrm{D}(\mathrm{X})$ corresponds to $\mathrm{X}$ exchanged hydrogen atoms] was observed with a corresponding decrease of the abundance of the precursor ion, $\mathrm{D}(0)$. The H/D exchange mass spectra after the reaction times of 0.01 , $2.5,40,120$, and $300 \mathrm{~s}$ are presented in Figure 1 for the three investigated oligonucleotides. A bimodal distribution is observed for $\mathrm{dTG}^{-}$and $\mathrm{C}_{6}{ }^{2-}$. It should be noted that no noticeable difference appeared in gas-phase $\mathrm{H} / \mathrm{D}$ exchange of $\mathrm{C}_{6}{ }^{2-}$ with or without $\mathrm{NH}_{4} \mathrm{Ac}$ in the injection solution. After $300 \mathrm{~s}$ of $\mathrm{H} / \mathrm{D}$ exchange, $\mathrm{dTG}^{-}$ exchanged mainly five of its six exchangeable hydrogens, and some totally deuterated ions were observed for $\mathrm{dC}_{6}{ }^{2-}$ (17 deuteriums) and $\mathrm{C}_{6}{ }^{2-}$ (23 deuteriums). It should be noticed that these latter situations still differ from the full $\mathrm{H} / \mathrm{D}$ exchange of the sample which would correspond to all ions being totally deuterated.

The rates of $H / D$ exchange reactions of ions that were accelerated upon entering the collision cell were also monitored. For a given H/D exchange time, the mass spectra obtained with and without ion acceleration were compared. No difference was observed for $\mathrm{dTG}^{-}$and $\mathrm{dC}_{6}{ }^{2-}$. But for $\mathrm{C}_{6}{ }^{2-}$, the bimodal profile was modified. The results are illustrated with ion acceleration at $20 \mathrm{~V}$ in Figure $2 \mathrm{a}$ and $\mathrm{b}$. With ion acceleration, the ratio of the two populations is altered; the abundance of the first population, which is associated with the less deuterated species, decreases, and the abundance of the second contribution, which is associated with the most deuterated species, increases. At a given reaction time, the maximum-minimum-maximum (M-m-M) positions, that characterize the positions and widths of the two contributions, are not affected by the acceleration step. However, these positions shift when the reaction time increases (Figure $2 b$ and $c$ ). In other words, acceleration and reaction time do not play the same role: a mass spectrum obtained with ion acceleration after a given reaction time will never be the same as a mass spectrum obtained without ion acceleration after a longer reaction time (Figure $2 \mathrm{a}$ and $\mathrm{c}$ ). Even after an exchange reaction time of $300 \mathrm{~s}$, ion acceleration keeps on influencing the ion distribution. Although the two contributions can no longer be distinguished, ion acceleration keeps on shifting the distribution more and more towards complete deuteration of the sample. 

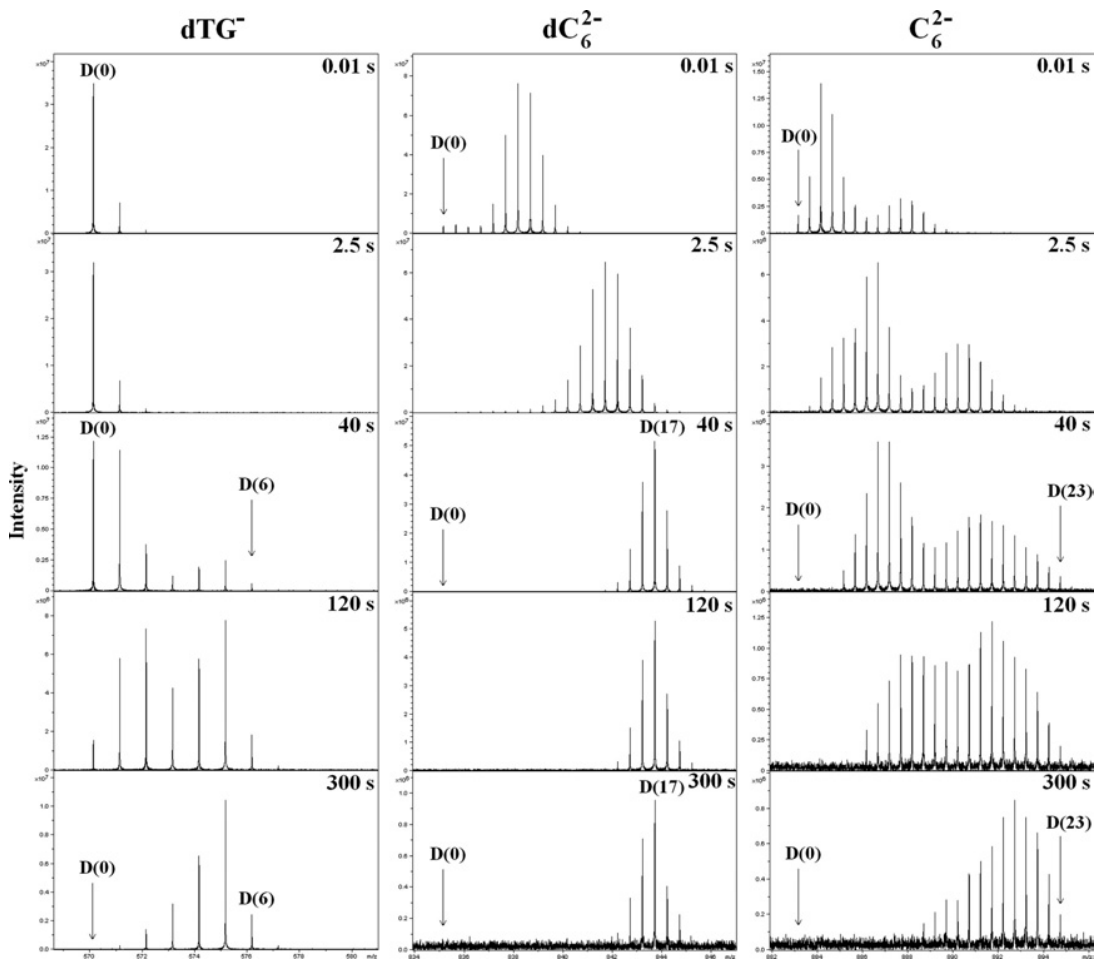

Figure 1. H/D exchange mass spectra at the reaction times of $0.01,2.5,40,120$, and $300 \mathrm{~s}$ for the three studied oligonucleotides.

As mentioned in the Experimental section, several values of the Col-Cell Trap parameter were tested for the $\mathrm{H} / \mathrm{D}$ exchange activation experiments. A modifica-

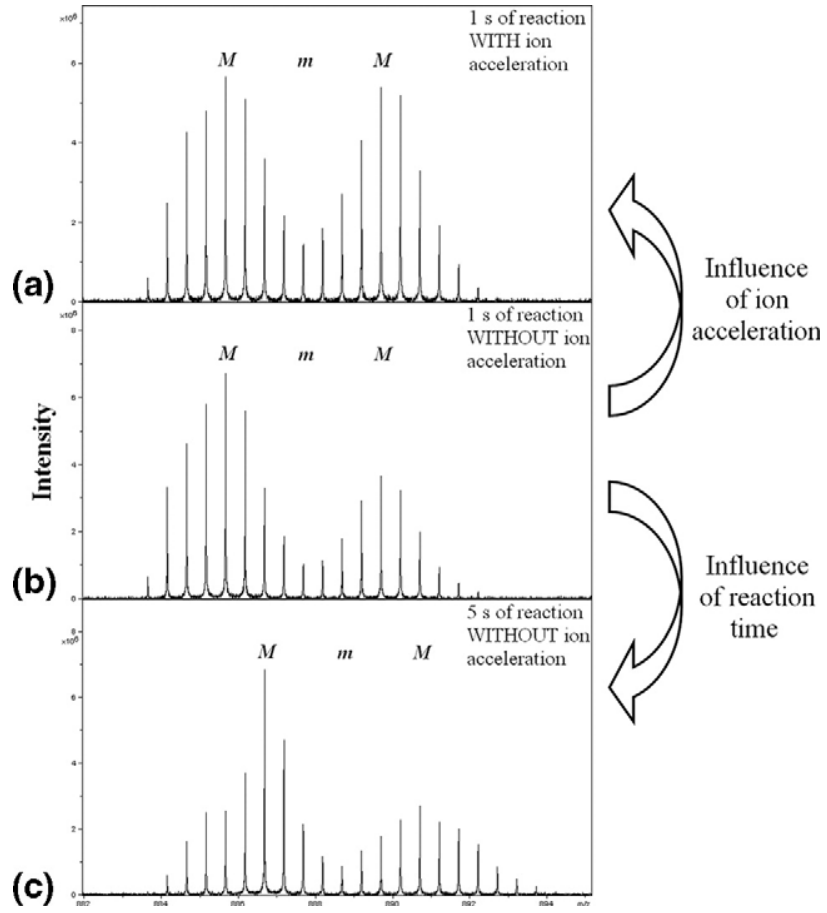

Figure 2. Comparison of $\mathrm{C}_{6}{ }^{2-} \mathrm{H} / \mathrm{D}$ exchange mass spectra recorded with and without ion acceleration; (a) $1 \mathrm{~s}$ of reaction with ion acceleration at $20 \mathrm{~V},(\mathbf{b}) 1 \mathrm{~s}$ of reaction without ion acceleration, and (c) $5 \mathrm{~s}$ of reaction without ion acceleration. tion of the $\mathrm{C}_{6}^{2-}$ bimodal profile (decrease of the abundance of the first contribution and increase of the abundance of the second one) was observed at each value. This ion distribution modification increased when the Col-Cell Trap parameter ranged from 7.5 to $15 \mathrm{~V}$, but leveled off from 15 to $20 \mathrm{~V}$.

For the analysis and the discussion of the previous results, reference will be made to results that were obtained during our previous study of the gas-phase $\mathrm{H} / \mathrm{D}$ exchange of dinucleotides [14]. In that work, dynamic simulations were carried out to complement the H/D exchange experiments, and to investigate the different conformations adopted by the dinucleotides. $\mathrm{dTG}^{-}$presents two distinctive structures that interconvert within the duration of a dynamic simulation (1 ns). The experimental kinetic plot of the $\mathrm{dTG}^{-} \mathrm{H} / \mathrm{D}$ exchange was approximated by adding the kinetic contributions of the two structures.

\section{Ion Mobility}

The recorded arrival time profiles (extracted mass mobilograms, stated in scans [27] are shown in Figure 3a-c for $\mathrm{dTG}^{-}, \mathrm{dC}_{6}{ }^{2-}$ and $\mathrm{C}_{6}{ }^{2-}$. $\mathrm{dTG}^{-}$and $\mathrm{dC}_{6}{ }^{2-}$ give rise to a single mobility peak. $\mathrm{C}_{6}{ }^{2-}$ shows a very wide drift time and its extracted mass mobilogram presents a composite mobility peak. A few experiments with ion acceleration in the source were carried out. No influence was observed for $\mathrm{dC}_{6}{ }^{2-}$, while the double mobility peak of $\mathrm{C}_{6}{ }^{2-}$ was affected as illustrated in Figure 4. The increase of the cone voltage induced an increase of the 

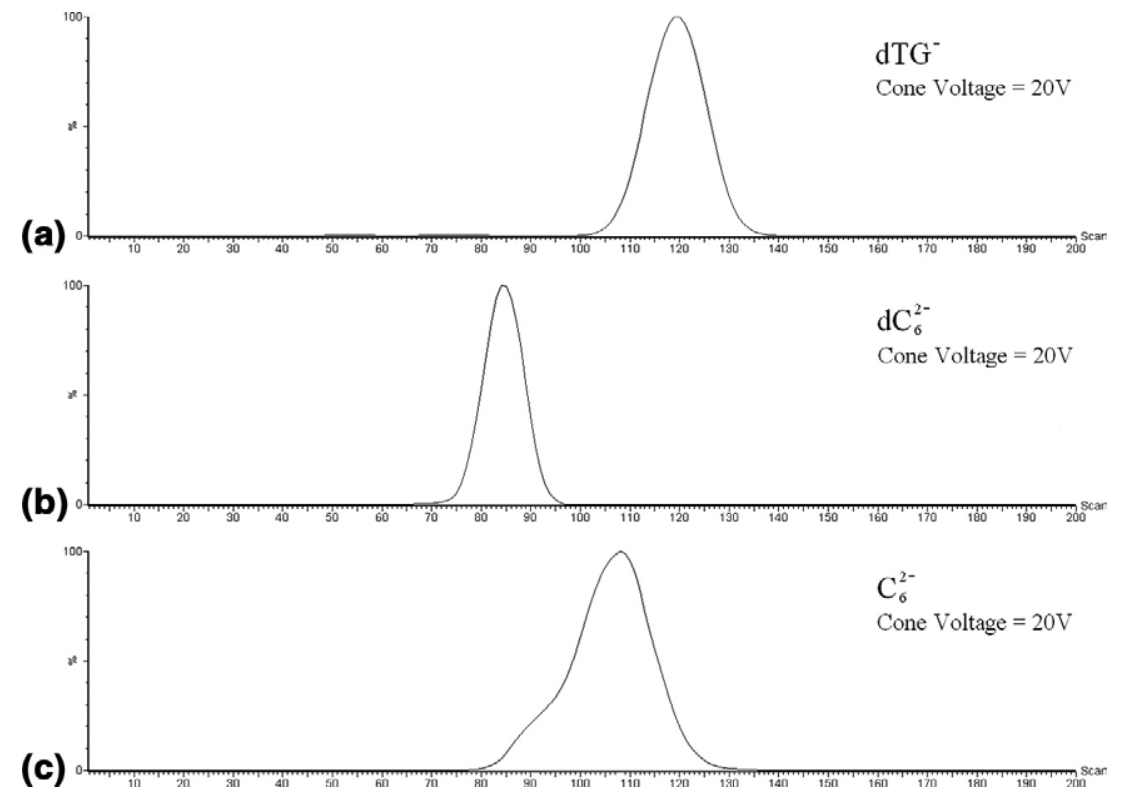

Figure 3. Mass mobilograms of the three studied oligonucleotides. (a) $\mathrm{dTG}^{-}$; (b) $\mathrm{dC}_{6}^{2-}$; (c) $\mathrm{C}_{6}^{2-}$.

short drift time contribution without affecting the drift times themselves (Figure 4 and Table 1). The mobilogram at $70 \mathrm{~V}$ is the same as the one at $50 \mathrm{~V}$ (Table 1).
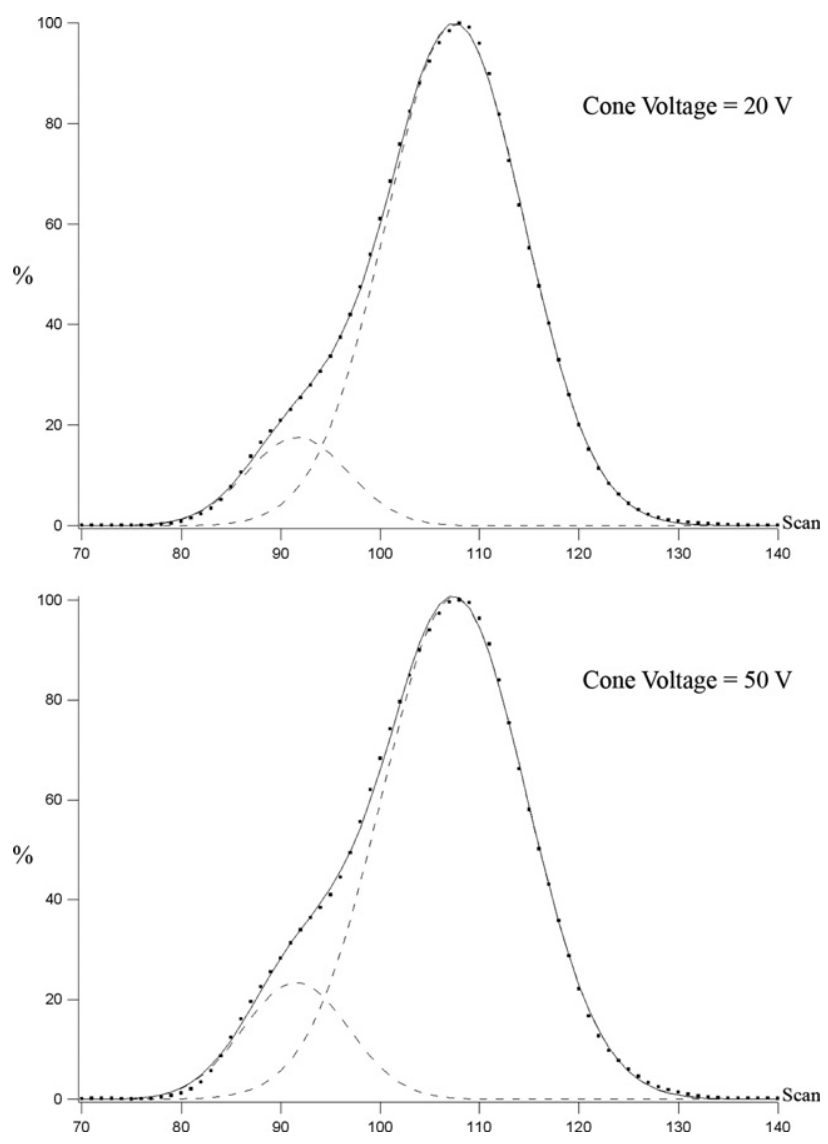

Figure 4. Comparison of $\mathrm{C}_{6}{ }^{2-}$ mass mobilograms recorded with and without ion activation. Dots: experimental data; dashed lines: individual Gaussian distributions; solid line: sum of these two Gaussian distributions.

\section{Discussion}

In our gas-phase H/D exchange study of dinucleotides, a kinetic model including simultaneous exchanges on the experimental time scale was proposed. In the framework of such a model, an observed bimodal exchange behavior can result not only from the presence of two gas-phase conformations with different exchange rates but also from the difference in accessibility of two $\mathrm{H}$ groups within a single structure. Indeed, by simulating H/D exchange kinetic plots (Matlab; The MathWorks Inc., Natick, MA) and by extracting from them the relative abundances of the deuterated species $[D(X)]$, bimodal profiles resulted from the presence of two groups of hydrogens within one structure (unpublished data). To distinguish experimentally between these two limit cases, it is necessary to combine complementary approaches:

1. The analysis of the influence of collisional activation on H/D exchange kinetics helps probing the height of the activation barriers connecting conformers and exchanged species.

2. Mobility measurements provides us with information on the presence of structures having different spatial extensions; recording how ion activation affects the mobility data makes the connection between both sets of experiments possible.

The aim of the present discussion is to present a model, described in Figure 5, which provides a consistent picture of the observed exchange and mobility data.

In the model, we have considered four stable species connected by activation barriers: two conformers and their respective perdeuterated species. It must be mentioned right away that this model simplifies the situation in at least two ways. First, each conformer is 
Table 1. Set of constants characterizing the two Gaussian distributions that were obtained using the procedure outlined in the text

\begin{tabular}{|c|c|c|c|c|c|c|}
\hline & \multicolumn{2}{|c|}{ Cone voltage $=20 \mathrm{~V}$} & \multicolumn{2}{|c|}{ Cone voltage $=50 \mathrm{~V}$} & \multicolumn{2}{|c|}{ Cone voltage $=70 \mathrm{~V}$} \\
\hline & $\begin{array}{l}\text { Short drift } \\
\text { time }\end{array}$ & $\begin{array}{l}\text { Long drift } \\
\text { time }\end{array}$ & $\begin{array}{c}\text { Short drift } \\
\text { time }\end{array}$ & $\begin{array}{l}\text { Long drift } \\
\text { time }\end{array}$ & $\begin{array}{l}\text { Short drift } \\
\text { time }\end{array}$ & $\begin{array}{l}\text { Long drift } \\
\text { time }\end{array}$ \\
\hline Area under the curve $\left(A_{i}\right)$ & $224 \pm 7$ & $1747 \pm 8$ & $311 \pm 12$ & $1838 \pm 12$ & $307 \pm 13$ & $1802 \pm 13$ \\
\hline Center of the curve $\left(n_{i}\right)$ & $91.7 \pm 0.2$ & $107.54 \pm 0.04$ & $91.5 \pm 0.2$ & $107.45 \pm 0.06$ & $93.2 \pm 0.2$ & $107.85 \pm 0.06$ \\
\hline Standard deviation $\left(\sigma_{\mathrm{i}}\right)$ & $5.1 \pm 0.1$ & $6.98 \pm 0.03$ & $5.3 \pm 0.1$ & $7.30 \pm 0.05$ & $5.8 \pm 0.1$ & $7.19 \pm 0.04$ \\
\hline Percentage of the Gaussian distribution & 11.4 & 88.6 & 14.5 & 85.5 & 14.5 & 85.5 \\
\hline
\end{tabular}

possibly representative for a group of conformers displaying close exchange rates and possibly low interconversion barriers. Second, the deuteration reaction is rather a multistep procedure with consecutive enthalpy and entropy bottlenecks. The different situations displayed in Figure 5a-c correspond to three limiting cases with, however, a constant feature: the $\mathrm{H} / \mathrm{D}$ exchange barriers can be overcome at room temperature on the experimental time scale. In Figure $5 \mathrm{a}$, a stable conformer is separated from a less stable one by a barrier exceeding the energy deposited upon activation. In Figure $5 \mathrm{~b}$, the isomerization barrier can be easily crossed at room temperature, while in Figure $5 c$, such a crossing is negligible and requires heating the system up using collisional activation. We now show that these three cases may correspond, respectively, to $\mathrm{dC}_{6}{ }^{2-}$, to $\mathrm{dTG}^{-}$, and to $\mathrm{C}_{6}{ }^{2-}$.

In the case of $\mathrm{dC}_{6}{ }^{2-}$, the mobility data (Figure $3 \mathrm{~b}$ ) identify only a single conformer (in the sense defined in the previous paragraph, i.e., possibly a group of conformers with similar properties). H/D exchange data are monomodal and remain unaffected upon activation. These observations are consistent with the situation depicted in Figure 5a except for the activation behavior where an apparent contradiction arises, which will be dismissed below. The largest stability of one conformer together with the high isomerization barrier prevents any other possible conformer to show up. The exchange rate, however, should be affected by the collisional heating, a prediction at odds with the experimental data (see the Results section). This behavior may result from two mechanisms. First the low H/D exchange activation barriers reduce the slope of the rate constant variation with temperature. Second and probably most important, fast collisional cooling takes place once acceleration is over. It is essential to remind here again that acceleration is limited to a short path at the entrance of the collision cell. The collisions that occur during this path are most probably only activating. Afterwards, the many collisions experienced by the ions within the collision cell lead to their being cooled down to room temperature, so that the effect of the activation process on the exchange rate is cancelled. It is hard to evaluate the duration of both processes (activation and cooling) but they are likely to be shorter that $0.01 \mathrm{~s}$ since no influence of the acceleration step is observed even at this shortest experimental time.
Figure $5 \mathrm{~b}$ accounts for the behavior of $\mathrm{dTG}^{-}$that displays a bimodal H/D exchange but a monomodal mobilogram and no influence of the activation step. The observation of a bimodal $\mathrm{H} / \mathrm{D}$ exchange and of a monomodal mobilogram can result as well from one conformer with two groups of exchangeable hydrogens as from two conformers that interconvert rapidly at room temperature. Neither H/D exchange experiments nor ion mobility measurements at room temperature can distinguish these two situations. Only a detailed structural study by molecular modeling [14] or ion mobility measurements at lower temperatures [21] can answer the question. These latter experiments led to the conclusion that the monomodal mobilogram results from the fast interconversion between the conformers separated by a small barrier, leading to an equilibrium between both species, and that the bimodal exchange pattern results from the different rates for both conformers, governed by their respective H/D exchange barriers. The absence of any activation signature is explained as in the previous paragraph by the deactivating collisions experienced by the trapped ions.

The third situation that we consider corresponds to an isomerization barrier that is higher than the exchange ones and that can be substantially overcome only after the ions have been activated (Figure $5 c$ ). This case corresponds to $\mathrm{C}_{6}{ }^{2-}$. Let us deal first with the nonactivated ions, i.e., with the room temperature situation. Due to the high barrier preventing fast isomerization, both conformers are present with proportions not necessarily in equilibrium and which depend in an unknown manner on the situation in solution and on the ionization mechanism. Each conformer exchanges $\mathrm{H}$ and $\mathrm{D}$ at its own rates, leading to a bimodal mass spectrum (Figure 1). In addition, the mobility data plead for two conformers with distinguishable spatial extensions (Figure 3c). Collisional activation increases the interconversion rate constants and leads to a new concentration ratio between both conformers that we assume now to be in equilibrium but that is eventually frozen when the ions are cooled down. This cooling process has the effect of reducing the isomerization rate constants to a point that the relaxation back to equilibrium is hindered on the experimental time scale. The system is left in a nonequilibrium situation where each conformer remains trapped in its own potential well. This is consistent with the observation that ion acceler- 
ation changes the ratio of the two contributions but not their positions because the exchange rates are kept unmodified. Moreover, because the population modifi-

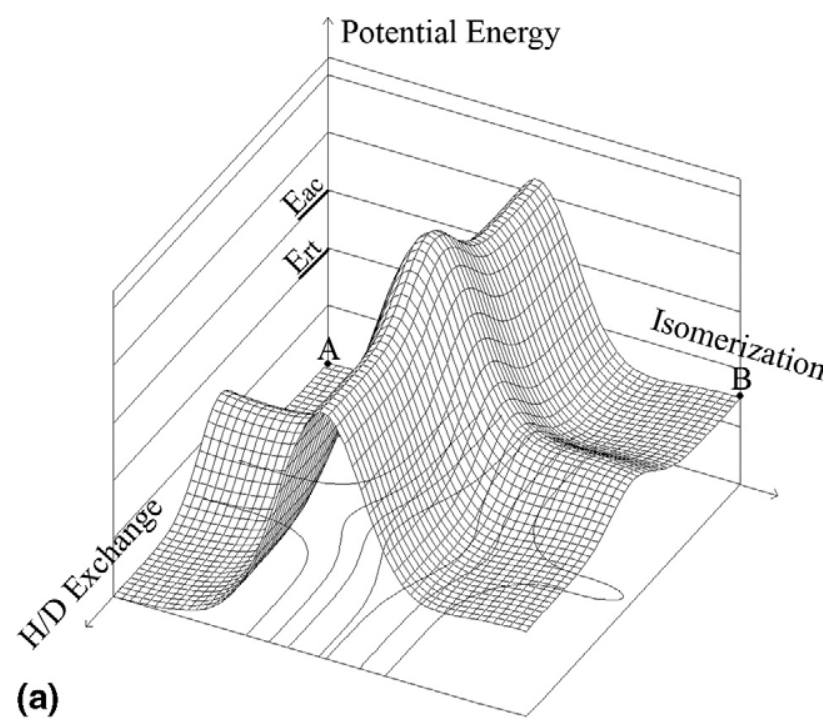

(a)

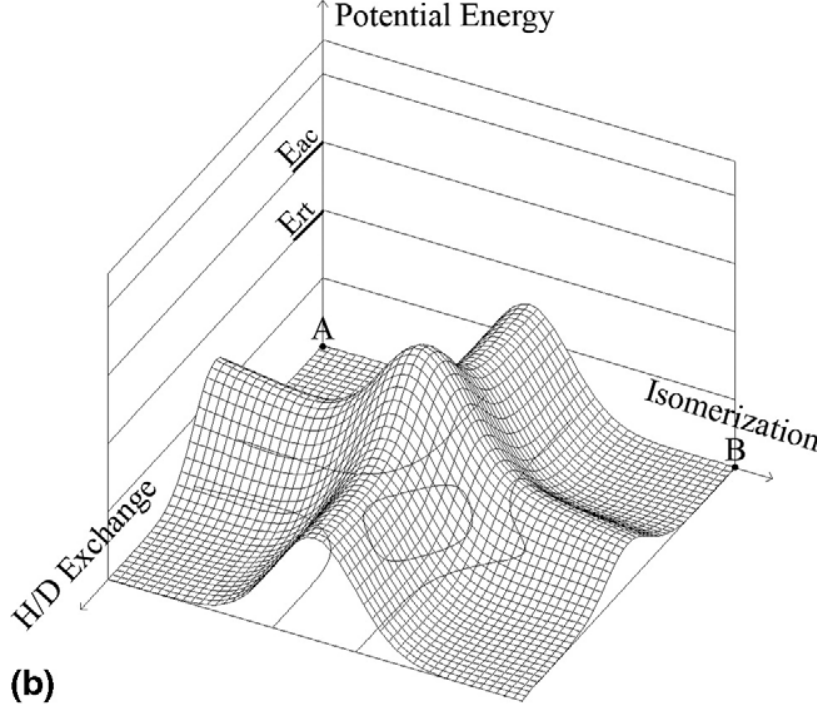

(b)

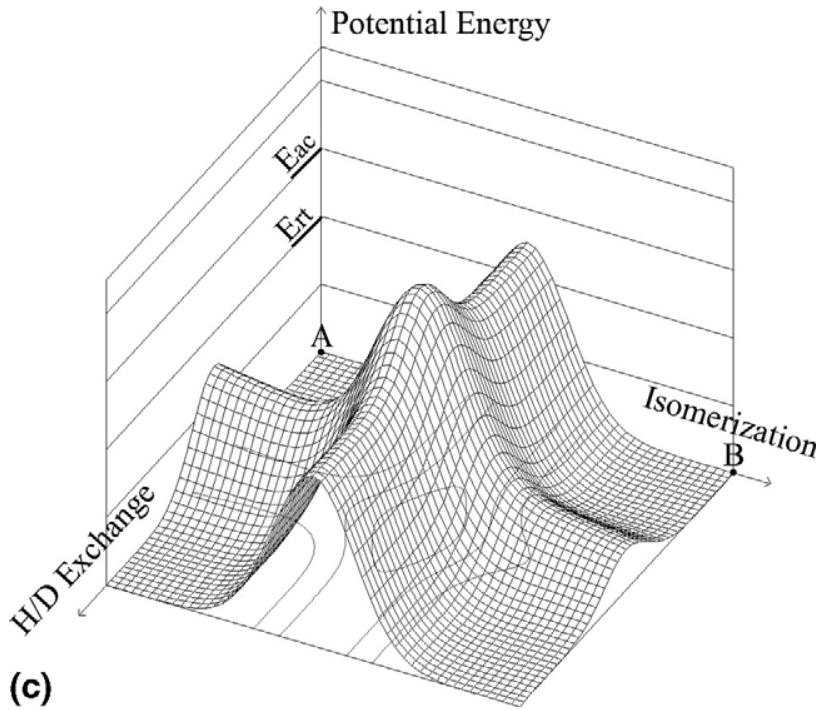

cation is frozen after the cooling process, the influence of ion acceleration is observed at each reaction time until complete exchange, where no difference should be observed. It is a signature of the activation process, which takes place during a very short time at the entrance of the collision cell. In a similar way as for $\mathrm{H} / \mathrm{D}$ exchange, $\mathrm{C}_{6}{ }^{2-}$ ion acceleration through the increase of the cone voltage changed the ratio of the two mobility peaks without affecting their drift times (Table 1, Figure 4); this is again in favor of a rapid cooling after the acceleration step.

It is observed that the concentration ratio of both conformers levels off between 50 and $70 \mathrm{~V}$. We propose the following tentative explanation. If we assume that the activation step leads to a sufficient increase of the isomerization rates for an equilibrium to obtain, this ratio is given by the equilibrium constant, i.e., $K=$ $e^{-\frac{\Delta_{r} G^{0}}{R T}}=e^{\frac{\Delta_{r} S^{0}}{R}} e^{-\frac{\Delta_{r} H^{0}}{R T}}$. Overcoming the high activation barrier requires depositing an amount of energy much larger than $\Delta_{\mathrm{r}} \mathrm{H}^{0}$. It has previously been shown that a temperature can usually be assigned to the activated ions and correlated to the cone voltage [28]. In other words, when the cone voltage is $\geq 50 \mathrm{~V}, \mathrm{RT} \gg \Delta_{\mathrm{r}} \mathrm{H}^{0}$ and $K \rightarrow e^{\frac{\Delta_{S} S 0}{R}}$. From the concentration ratio at 50 and 70 $\mathrm{V}, \Delta_{\mathrm{r}} \mathrm{S}^{0}=-14.8 \mathrm{~J} \mathrm{~mol}^{-1} \mathrm{~K}^{-1}$ can be estimated, a value consistent with a smaller entropy for the more compact structure. The fact that in both H/D exchange and ion mobility experiments the results tend to level off when the voltage is increased is an indication that an equilibrium distribution results from the activation step.

The $\mathrm{C}_{6}{ }^{2-}$ conformer whose proportion increases upon ion acceleration is the one that exchanges the largest number of hydrogens and that has the most compact structure (Figure 2 and Figure 4). Other studies have reported identical observations of compact structures (small cross section) with fast H/D exchange [5, 17]. Although it is intuitively surprising, it is conceivable that a compact structure possesses exchangeable hydrogens with high $\mathrm{H} / \mathrm{D}$ exchange rate constants. To account for these high rate constants, direct accessibility of hydrogens or more probably various intramolecular "relay" mechanisms (not to be confused with the relay mechanism that is mentioned in the mononucleotides studies [7-11]), which can bring deuteriums into the compact structure, and/or H/D exchange barriers changes upon folding can be evoked. A detailed structural study of $\mathrm{C}_{6}{ }^{2-}$ searching for different stable struc-

Figure 5. Proposed model. $\mathrm{E}_{\mathrm{ac}}$ is the internal energy of the ion reached during the ion acceleration process. $E_{r t}$ is the internal energy of the ion at room-temperature. The H/D exchange and isomerization nuclear coordinates are assumed to be independent. The two conformers, A and B, possess different H/D exchange properties. Although the relative energies have a significant impact on the conformer abundances, the two conformers are represented, in the situations (b) and (c), with identical zero-point energies to simplify the model and to make the picture more readable. 
tures and possibly comparing the theoretical and experimental cross sections would be very relevant. Even if this study is time-consuming due to the complexity and the large number of possible relevant conformations of $\mathrm{C}_{6}{ }^{2-}$, it is planned and will hopefully be presented in a forthcoming publication.

The observation of a compact structure with a fast $H / D$ exchange shows that the H/D exchange and ion mobility methods are not directly comparable but are complementary as accessibility and compactness are connected to different physical properties. In addition, both methods probe different timescales. In summary, $\mathrm{H} / \mathrm{D}$ exchange can resolve different families of conformers that cannot be resolved using ion mobility and vice versa [5]. An ion could display a monomodal H/D exchange and be detected as a double mobility peak, and vice versa.

\section{Conclusions}

In this work, gas-phase $\mathrm{H} / \mathrm{D}$ exchange is shown as an efficient tool for detecting the presence of one structure (or one group of structures having similar exchange rates) or the presence of several conformers (or groups of conformers) that possess different exchange properties. Moreover, if several structures are observed, increasing the internal energy of the ions at the entrance of the H/D exchange cell gives access to a qualitative estimation of the relative height of the isomerization barrier compared with the H/D exchange barriers. Ion mobility experiments can confirm the H/D exchange results on the basis of independent data. The ion mobility experiments allow us to distinguish between two groups of situations, indeed: (1) on the one hand, only one structure (or one group of structures having the same compactness) is present or several conformers exist but interconvert rapidly at the experimental temperature; (2), on the other hand, several conformers are present and do not interconvert. In the present study, the $\mathrm{H} / \mathrm{D}$ exchange results for $\mathrm{C}_{6}{ }^{2-}$ are confirmed by ion mobility experiments both for the presence of two gasphase conformations, and for their non-interconversion at room temperature. By comparing the ion acceleration experiments for $\mathrm{H} / \mathrm{D}$ exchange and for ion mobility, the $\mathrm{C}_{6}{ }^{2-}$ conformer that possesses the fastest $\mathrm{H} / \mathrm{D}$ exchange is identified as the one having the smallest measured drift time and therefore the smallest collision cross section. The hydrogen accessibility and the compactness are structural properties that are connected to different physical parameters. The compactness of the structure and its H/D exchange rates cannot be analyzed with only one of the two methods. For the study of biomolecule gas-phase conformations, the combination of $\mathrm{H} / \mathrm{D}$ exchange and ion mobility provides us with extra information compared with the two individual methods used alone.

\section{Acknowledgments}

The authors acknowledge support for this work by the FRS-FNRS (Fonds de la Recherche Scientifique-FNRS), Belgium.

\section{References}

1. Gard, E.; Green, M. K.; Bregar, J.; Lebrilla, C. B. Gas-Phase Hydrogen/ Deuterium Exchange as a Molecular Probe for the Interaction of Methanol and Protonated Peptides. J. Am. Soc. Mass Spectrom. 1994, 5, 623-631.

2. Campbell, S. C.; Rodgers, M. T.; Marzluff, E. M.; Beauchamp, J. L. Deuterium Exchange Reactions as a Probe of Biomolecule Structure. Fundamental Studies of Gas Phase H/D Exchange Reactions of Protonated Glycine Oligomers with $\mathrm{D}_{2} \mathrm{O}, \mathrm{CD}_{3} \mathrm{OD}, \mathrm{CD}_{3} \mathrm{CO}_{2} \mathrm{D}$, and $\mathrm{ND}_{3}$. J. Am. Chem. Soc. 1995, 117, 12840-12854.

3. Wang, F.; Freitas, M. A.; Marshall, A. G.; Skyes, B. D. Gas-Phase Memory of Solution-Phase Protein Conformation: H/D Exchange and Fourier Transform Ion Cyclotron Resonance Mass Spectrometry of the N-terminal Domain of Cardiac Troponin C. Int. J. Mass Spectrom. 1999, 192, 319-325.

4. Herrmann, K. A.; Kuppannan, K.; Wysocki, V. H. Fragmentation of Doubly-Protonated Peptide Ion Populations labeled by H/D Exchange with $\mathrm{CD}_{3} \mathrm{OD}$. Int. J. Mass Spectrom. 2006, 249/250, 93-105.

5. Robinson, E. W.; Williams, E. R. Multidimensional Separations of Ubiquitin Conformers in the Gas Phase: Relating Ion Cross Sections to H/D Exchange Measurements. J. Am. Soc. Mass Spectrom. 2005, 16, 1427-1437.

6. Sawyer, H. A.; Marini, J. T.; Stone, E. G.; Ruoloto, B. T.; Gillig, K. J.; Russell, D. H. The Structure of Gas-Phase Bradykinin Fragment 1-5 (RPPGF) Ions: An Ion Mobility Spectrometry and H/D Exchange Ion-Molecule Reaction Chemistry Study. J. Am. Soc. Mass Spectrom. 2005, 16, 893-899.

7. Freitas, M. A.; Shi, S. D. H.; Hendrickson, C. L.; Marshall, A. G. Gas-Phase RNA and DNA Ions. 1. H/D Exchange of the $[\mathrm{M}-\mathrm{H}]^{-}$Anions of Nucleoside 5'-Monophosphates (GMP, dGMP, AMP, dAMP, CMP, dCMP, UMP, dTMP), Ribose 5-Monophosphate, and 2-Deoxyribose 5-Monophosphate with $\mathrm{D}_{2} \mathrm{O}$ and $\mathrm{D}_{2} \mathrm{~S}$. J. Am. Chem. Soc. 1998, 120, 1018710193.

8. Robinson, J. M.; Greig, M. J.; Griffey, R. H.; Venkantraman, M.; Laude, D. A. Hydrogen/Deuterium Exchange of Nucleotides in the Gas Phase. Anal. Chem. 1998, 70, 3566-3571.

9. Freitas, M. A.; Marshall, A. G. Gas-Phase RNA and DNA Ions. 2. Conformational Dependence of the Gas-Phase H/D Exchange of Nucleotide-5'-Monophosphates. J. Am. Soc. Mass Spectrom. 2001, 12, 780-785

10. Crestoni, M. E.; Fornarini, S. Gas-Phase Hydrogen/Deuterium Exchange of Adenine Nucleotides. J. Mass Spectrom. 2003, 38, 854-861.

11. Chipuk, J. E.; Brodbelt, J. S. Gas-Phase Hydrogen/Deuterium Exchange of 5'- and 3'-Mononucleotides in a Quadrupole ion Trap: Exploring the Role of Conformation and System Energy. J. Am. Soc. Mass Spectrom. 2007, 18, 724-736.

12. Felix, T.; Reyzer, M.; Brodbelt, J. Hydrogen/Deuterium Exchange of Nucleoside Analogs in a Quadrupole ion Trap Mass Spectrometer. Int. J. Mass Spectrom. 1999, 191, 161-170.

13. Gree-Church, K. B.; Limbach, P. A.; Freitas, M. A.; Marshall, A. G. Gas-Phase Hydrogen/Deuterium Exchange of Positively Charged Mononucleotides by Use of Fourier-Transform Ion Cyclotron resonance Mass Spectrometry. J. Am. Soc. Mass Spectrom. 2001, 12, 268-277.

14. Balbeur, D.; Dehareng, D.; De Pauw, E. Conformationally Driven Gas-Phase H/D Exchange of Dinucleotide Negative Ions. J. Am. Soc. Mass Spectrom. 2007, 18, 1827-1834.

15. Hofstadler, S. A.; Griffey, R. H. Analysis of Noncovalent Complexes of DNA and RNA by Mass Spectrometry. Chem. Rev. 2001, 101, 377-390.

16. Mo, J.; Hakansson, K. Oligonucleotide Gas-Phase Hydrogen/Deuterium Exchange with $\mathrm{D}_{2} \mathrm{~S}$ in the Collision Cell of a Quadrupole-Fourier Transform Ion Cyclotron Resonance Mass Spectrometer. Anal. Chem. 2007, 79, 7893-7898.

17. Gabelica, V.; Rosu, F.; Witt, M.; Baykut, G.; De Pauw, E. Fast Gas-Phase Hydrogen/Deuterium Exchange Observed for a DNA G-Quadruplex. Rapid Commun. Mass Spectrom. 2005, 19, 201-208.

18. Griffey, R. H.; Greig, M. J.; Robinson, J. M.; Laude, D. A. Gas-Phase Hydrogen-Deuterium Exchange in Phosphorothioate d(GTCAG) and d(TCGAT). Rapid Commun. Mass Spectrom. 1999, 13, 113-117.

19. Gidden, J.; Bowers, M. T. Gas-Phase Conformation of Deprotonated and Protonated Mononucleotides Determined by Ion Mobility and Theoretical Modeling. J. Phys. Chem. 2003, 107, 12829-12837.

20. Liu, D.; Wyttenbach, T.; Bowers, M. T. Hydration of Mononucleotides. J. Am. Chem. Soc. 2006, 128, 15155-15163.

21. Gidden, J.; Bowers, M. T. Gas-Phase Conformational and Energetic Properties of Deprotonated Dinucleotides. Eur. Phys. J. D. 2002, 20, 409-419.

22. Gidden, J.; Bowers, M. T. Gas-Phase Conformations of Deprotonated Trinucleotides $\left(\mathrm{dGTT}^{-}, \mathrm{dTGT}^{-}\right.$, and $\left.\mathrm{dTTG}^{-}\right)$: The Question of Zwitterion Formation. J. Am. Soc. Mass Spectrom. 2003, 14, 161-170. 
23. Hoaglund, C. S.; Liu, Y.; Ellington, A. D.; Pagel, M.; Clemmer, D. E. Gas-Phase DNA: Oligothymidine Ion Conformers. J. Am. Chem. Soc. 1997, 119, 9051-9052.

24. Gidden, J.; Baker, E. S.; Ferzoco, A.; Bowers, M. T. Structural Motifs of DNA Complexes in the Gas Phase. Int. J. Mass Spectrom. 2005, 240, 183-193.

25. Baker, E. S.; Bernstein, S. L.; Gabelica, V.; De Pauw, E.; Bowers, M. T. G-Quadruplexes in Telomeric Repeats are Conserved in a Solvent-Free Environment. Int. J. Mass Spectrom. 2006, 253, 225-237.

26. Gabelica, V.; Baker, E. S.; Teulade-Fichou, M.-P., De Pauw, E.; Bowers, M. T. Stabilization and Structure of Telomeric and c-myc Region
Intramolecular G-Quadruplexes: The Role of Central Cations and Small Planar Ligands. J. Am. Chem Soc. 2007, 129, 895-904.

27. Pringle, S. D.; Giles, K.; Wildgoose, J. L.; Williams, I. P.; Slade, S. E.; Thalassinos, K.; Bateman, R. H.; Bowers, M. T.; Scrivens, J. H. An Investigation of the Mobility Separation of Some Peptide and Protein Ions Using a New Hybrid Quadrupole/Traveling Wave IMS/oa-TOF instrument. Int. J. Mass Spectrom. 2007, 261, 1-12.

28. Hoxha, A.; Collette, C.; De Pauw, E.; Leyh, B. Mechanism of Collisional Heating in Electrospray Mass Spectrometry: Ion Trajectory Calculations. J. Phys. Chem. A 2001, 105, 7326-7333. 